\title{
ANALYSIS OF TRUNK POWER AND JOINT STRESSES BETWEEN PROFESSIONAL AND COLLEGIATE PITCHERS
}

\author{
Maxwell L Albiero, Cody Dziuk, and Janelle A Cross
}

Department of Orthopaedic Surgery, Medical College of Wisconsin, Milwaukee, Wisconsin, USA

Corresponding Author: Maxwell L Albiero

Email: malbiero@mcw.edu

https://doi.org/10.34107/BiomedSciInstrum.57.04121

\begin{abstract}
The dynamic motion of a baseball pitch generates high elbow and shoulder torques that can result in injury. Previous research has noted the importance of properly transferring energy from the lower extremities through the throwing arm to decrease joint stress. The goal of this study was to compare segmental powers between two levels of pitchers at various moments throughout the pitching cycle and observe their influence on upper extremity torques. Thirteen professional and thirteen collegiate pitchers participated in this study. Forty-seven reflective markers were attached to the subjects at specific landmarks. An 8-camera motion analysis system was set up surrounding an artificial pitching mound, where participants threw 10 fastballs. Data were exported and processed using Visual 3D software. Welch's T-tests compared the means between groups with a significance set at $\mathrm{p}<0.05$. Professional pitchers were found to have significantly greater torso power at foot contact, maximum shoulder external rotation, ball release, and overall peak torso power. They also demonstrated significantly greater pitch velocity. Professional pitchers generated similar elbow varus torque and shoulder internal rotation torque compared to collegiate pitchers. These findings suggest professional pitchers more effectively use torso power to help increase pitch speed without increasing overall joint torques.
\end{abstract}

Keywords: biomechanics, kinetics, elbow torque, energy flow

\section{INTRODUCTION}

Elbow and shoulder injury often occur in baseball pitchers due to overuse. The repetitive throwing motion creates microtrauma in the musculoskeletal system that is unable to fully recover [1]. Previous research has found pitchers can generate forces five times beyond the athletes body weight [2]. These forces exceed surrounding joint ligament and muscle physiological thresholds increasing risk for injury. Specifically, injury to the elbow joint has been shown to be at significantly higher risk than other regions of the body due to elbow valgus overloading [3]. It has previously been shown that continuous medial elbow valgus overloading can lead to ulnar collateral ligament injury (UCL) [4], and that there has been a steady increase in UCL injury across all levels, leading to $25 \%$ of Major League Baseball (MLB) pitchers having reported suffering the injury [5].

During the pitching cycle (PC), energy is generated from the force of the back leg and other various biomechanical variables through sequential body motion. Studies have supported the notion that this transfer of segmental energy generally follows a proximal-to-distal flow through the throwing arm, from the hips to the forearm, creating a biomechanical chain of forces and torques [3,6,7]. Monitoring how this energy courses through the body during the pitching motion with the use of three-dimensional (3D) motion analysis on individual body segments can lead to a stronger understanding of the creation of excessive forces on the elbow and shoulder, and more importantly, how they may be prevented to avoid injury. Previous research has suggested that due to the large segmental mass, the trunk may be a primary contributor to the work of the throwing arm [8]. Additionally, a research study conducted by Aguinaldo et al found results demonstrating correlations in biomechanical variables between baseball pitchers of different levels with joint torques [7]. Therefore, the purpose of 